\title{
Serum demyelinating factors and adjuvant-like activity of Mycobacterium leprae: possible causes of early nerve damage in leprosy
}

\author{
V P SHETTY, N F MISTRY \& N H ANTIA \\ The Foundation for Medical Research 84-A, R.G. Thadani Marg, \\ Worli, Bombay-400 018
}

\begin{abstract}
Accepted for publication 4 December 1984
Summary The role of antibody was investigated by (a) subcutaneous injection using whole serum obtained from 7 leprosy patients, and (b) intraneural injection using immunoglobulins from 8 randomly chosen leprosy patients in random bred Swiss white mice. Three out of 15 samples showed positive demyelination.

The adjuvant like activity of Mycohacterium leprae was studied for its role in the causation of primary nerve damage of Swiss white mice. Animals were injected subcutaneously with $20 \times 10^{6}$ live and heat killed $M$. leprae with and without normal sciatic nerve extract. Biopsies of the sciatic nerves were performed 1 and 3 months after injection. Degenerative changes in the non-myelinated fibres were observed at the first month in the sciatic nerves of mice injected with live and heat-killed $M$. leprae along with nerve extract.
\end{abstract}

\section{Introduction}

Our previous studies on early leprosy cases, contacts of leprosy patients and sciatic nerves of foot-pad inoculated mice revealed a characteristic sequence of nerve damage which was typical of leprosy. Such pathological changes were observed in nerves remote from the site of infection even in the absence of acid fast bacilli or inflammatory cells. ${ }^{1-3}$.

This raised the possibility of the presence of circulating factors directed against nerve components. Such a hypothesis was consistent with the fact that Mycobacteria exerting adjuvant like action gave rise to auto-antibodies. ${ }^{4}$

Our objectives in Part I of this study were therefore to detect serum demyelinating factors, if any, in leprosy patients. Part II of this study consisted of assessing changes in mouse sciatic nerve that could be brought about by a subcutaneous injection of normal mouse sciatic nerve extract and $M$. leprae. 


\section{Materials and methods}

\section{PART I SERUM DEMYELINATION STUDIES}

\section{(a) Subcutaneous injection using whole serum}

Sera from 7 randomly chosen leprosy patients (4BT, 3BL) were injected subcutaneously into the right and left flanks above the thigh region of normal, random bred Swiss white mice (6 weeks old) in a total dosage of $0.5 \mathrm{ml}$. Each sample was injected into 2 mice. Biopsy of both the sciatic nerves was performed 1 month after the injection.

\section{(b) Intraneural injection using immunoglobulin}

In another series of experiments, immunoglobulins obtained by ammonium sulphate precipitation from sera of 8 randomly selected leprosy patients (4BT, $4 \mathrm{BL}$ ) were injected intraneurally into the right sciatic nerves of normal Swiss white mice in a dosage of $0.5 \mu \mathrm{l}$ using a glass micropipette. Biopsies of both the sciatic nerves were collected at the end of 1 week.

The binding of antibody to nerve was also checked in vitro using an indirect fluorescent system consisting of sequential application of test serum and FITC labelled Protein A.

Subcutaneous and intraneural injection of human $\mathrm{AB}$ serum and normal saline served as controls.

PART II ADJUVANT LIKE ACTIVITY OF M. LEPRAE

The experiments were designed as indicated in Table 1.

Table 1. Various combinations of inocula and the sciatic nerve biopsy interval in the mice

Number of mice studicd at

Inocula

1st month 3rd month

\begin{tabular}{llc}
\hline A $25 \times 10^{6}$ viable $M$. leprae & 2 & $\mathrm{Nil}$ \\
B $25 \times 10^{6}$ heat killed $M$. leprae & 2 & $\mathrm{Nil}$ \\
C $25 \times 10^{6}$ viable $M$. leprae $+10 \%$ nerve extract in PBS & 2 & $(2+2)^{*}$ \\
D $25 \times 10^{6}$ heat killed $M$. leprae $+10 \%$ nerve extract & 2 & $(2+2)^{*}$ \\
E Nerve extract alone $(10 \%$ in PBS) & 2 & $\mathrm{Nil}$ \\
\hline
\end{tabular}

* Two mice for sciatic nerve biopsy and 2 mice used for collection of blood. 
$M$. leprae was derived from homogenized skin of untreated bacilliferous patient. Inocula of $0.1 \mathrm{ml}$ containing $25 \times 10^{6} \mathrm{M}$. leprae with or without nerve extract, was injected subcutaneously into one flank of random bred Swiss white mice (1-2 months old). For each set of experiments the same batch of infecting organism was used.

To detect serum demyelinating factors, serum was collected by cardiac puncture at the 3 month period from 2 animals injected with $25 \times 10^{6}$ viable $M$. leprae $+10 \%$ nerve extract in PBS and 2 animals injected with $25 \times 10^{6}$ heat-killed $M$. leprae $+10 \%$ nerve extract in PBS (Inocula C \& D in Table 1). Each serum sample was injected intraneurally as previously described in the right sciatic nerves of 2 mice.

Sciatic nerve biopsies of both parts of this study were processed for electron microscopy. The sections were examined primarily at the semi-thin level and wherever necessary at the ultra-thin level using a JEOL 100S TEM.

\section{Observations}

\section{PART I SERUM DEMYELINATION STUDIES}

(a) In the first set of experiments where whole serum was used, 1 (from an untreated BT patient) out of 7 serum samples produced patchy segmental demyelination of $20-30 \%$ of fibres (Figure $1(\mathrm{a})$ and (b)) in all the 4 nerves obtained from 2 mice. The changes were predominantly perivascular. These mice had also shown signs of hind leg weakness.

(b) In the second series 2 out of 8 samples revealed similar patchy demyelination in the injected sciatic nerves. No pathology was seen in the contralateral uninjected nerve of the same animal. Of the two positive samples one belonged to a borderline lepromatous bacillary skin negative patient treated for 16 years; the second was obtained from a bacillary positive borderline lepromatous patient treated for 2 years.

Serum from the above mentioned BT patient and the latter BL patients showed positive binding activity to normal mouse nerve by fluorescence.

In the control group no demyelinating activity was detected in subcutaneous injections, however in intraneural inoculations, mild axonal degeneration was noted at the site of injection.

\section{PART II ADJUVANT LIKE ACTIVITY OF $M$. LEPRAE}

A large subcutaneous dose of $25 \times 10^{6}$ viable and heat-killed $M$. leprae (inocula A $\&$ B, Table 1) did not reveal any pathological changes in the sciatic nerves of mice at 1 month. However in combination with normal mouse sciatic nerve extract (inocula $C \& D$ ) significant degenerative changes were seen in the unmyelinated 

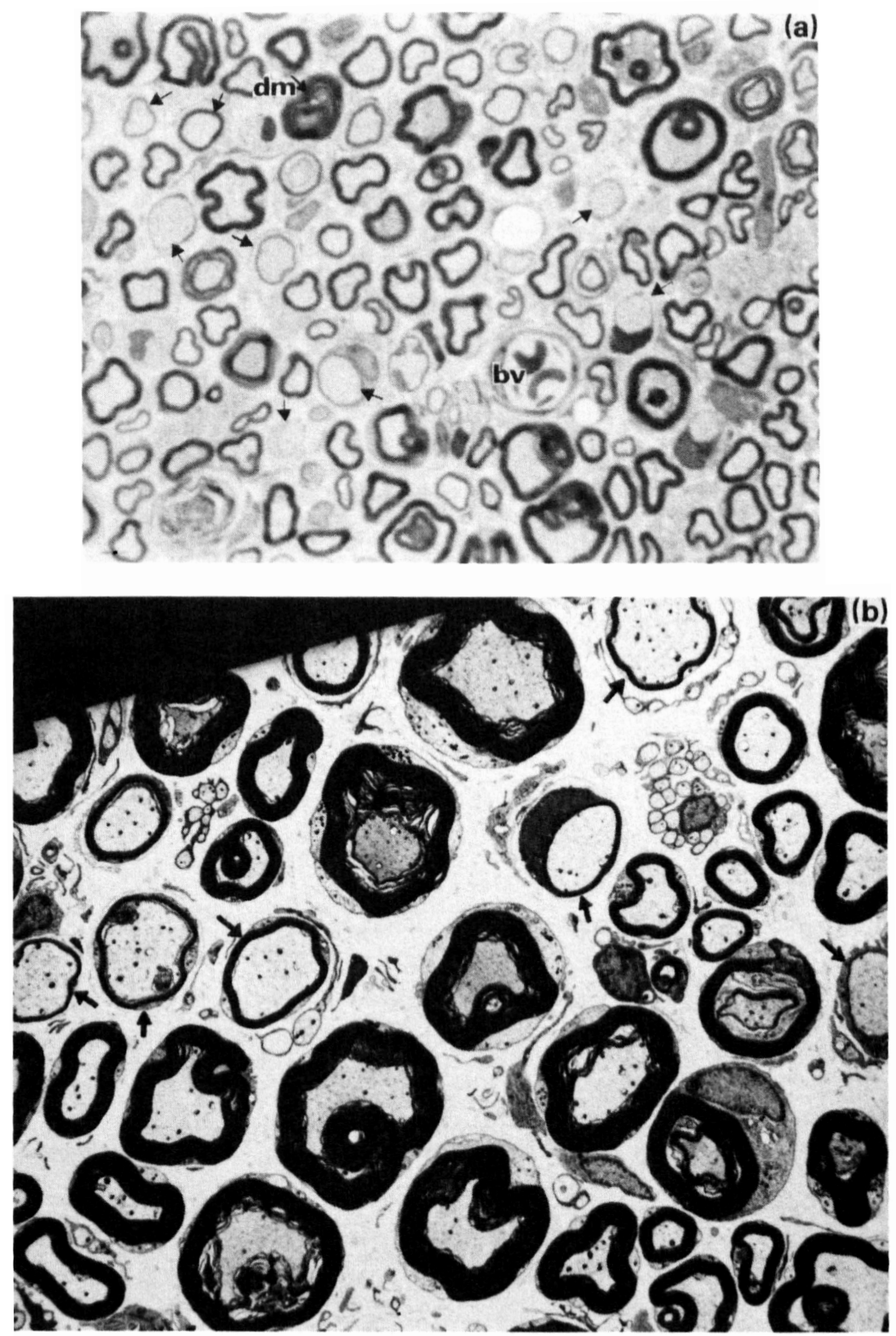

Figure 1. (a) Demyelination of fibres seen in the sciatic nerve of a mouse injected subcutaneously with $0.5 \mathrm{ml}$ of serum obtained from an untreated borderline tuberculoid patient. Biopsy was obtained 1 month after injection. Number of demyelinated axons (arrows) are seen. There is good clearance of myelin debris and most of the fibres show thin rim of myelin (arrow heads) suggesting remyelination. One micron thick, transverse semi-thin section stained with toludine blue. dm, degenerated myelin; bv, blood vessel.

(b) Part of the nerve shown in (a) at ultrastructural level showing a number of demyelinated fibres (arrows) with remyelination. $\times 2000$. 
fibres (Figure 2). Contrary to expectations the damage did not extend to myelinated fibres at 3 months; however the initial changes in non-myelinated fibres persisted although to a lesser degree.

Serum obtained at 3 months from the mice injected with inocula C \& D failed to demonstrate any demyelinating activity when injected intraneurally into normal Swiss white mice sciatic nerves. Nerve extract (inoculum E) by itself was also unable to induce such changes.

\section{Discussion}

Since mycobacteria are known to exert adjuvant like activity ${ }^{5}$ it is expected that in a disease like leprosy, autoimmune reactions to peripheral nerve components could be generated. Since myelin basic protein (MBP) is known as a potent antigen, one would favour it as the target component. However, a recent study ${ }^{6}$ and an earlier one ${ }^{7}$ could not trace any anti-MBP antibodies in leprosy patients; the latter group however recorded the presence of anti-axonal antibodies.

The first part of our study detects a demyelinating antibody, though its exact specificity remains unknown. Antibody mediated demyelination analogous to Experimental Allergic Neuritis (EAN) was reported ${ }^{8}$ for the first time without the use of adjuvants via intraneural injection of EAN serum. Using this same

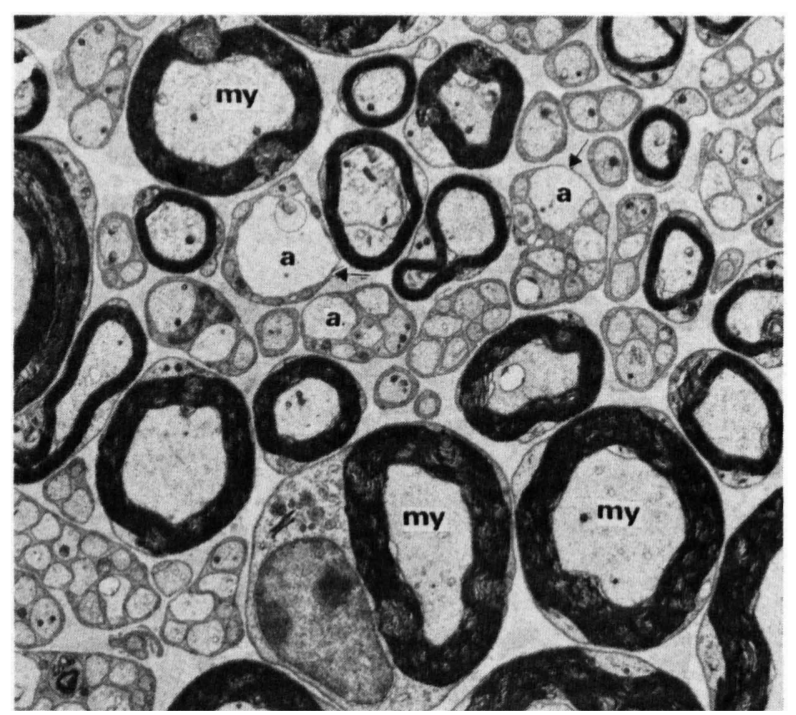

Figure 2. Part of the sciatic nerve from a mouse injected subcutaneously with $20 \times 10^{6}$ viable $M$. leprae $+10 \%$ whole nerve extract. Biopsy obtained after 1 month. Number of unmyelinated axons (a) shows degenerative changes like disintegration of neurotubules and filament giving watery appearance to the axoplasm. There is also abnormal Schwann cell axon contact (arrows). Myelinated fibres (my) are well preserved. Electron micrograph. $\times 4000$. 
technique we were successful in transmitting demyelinating factor from the patient's serum to a normal mouse in the absence of any presensitizing event.

In the subcutaneously injected mice demyelination was seen predominantly in the perivascular regions suggesting that the factor could have leaked through the blood vessels. The results implied that disseminated neural damage could occur in spite of localized infection and could persist even after prolonged therapy. It would be interesting, however, to know whether demyelination involves the participation of any inflammatory cells and secondly whether it is a single stage process. These observations may also explain the early pathological changes of leprosy patients in nerves as described previously by us. ${ }^{1,2}$ The low frequency of finding a serum factor positive for demyelinating activity may be attributed to catabolic processes and periodic variation in serum antibody concentration.

Our results in the second series of experiments confirm the postulates of another study, ${ }^{9}$ that the earliest change in leprous neuropathy is due at least partly to an autoimmune reaction against neural antigens. Unlike their experiments where granulomatous hypersensitivity was manifested in cutaneous nerves, our experiments using $M$. leprae as adjuvant demonstrated degenerative changes in the nonmyelinated fibres in a major nerve trunk away from the site of inoculation. It was interesting to note that these changes were identical to those seen in the unmyelinated fibres of foot-pad inoculated mice between 4 th and 8 th post-inoculation month. ${ }^{3}$

The two interesting features of this early change are that it requires no presensitization for its expression nor is it necessarily linked to sequential segmental demyelination. The inability to detect progressive nerve damage at 3 months is hard to explain. Perhaps it is a dose effect of the concentration of $M$. leprae/sciatic nerve extract used; hypersensitivity being brought on with larger doses. Segmental demyelination and ensuing inflammation can perhaps occur only when there is systemic sensitization of $M$. leprae leading to a break in the blood nerve barrier. ${ }^{10}$

\section{References}

I Shetty VP, Mehta LN, Irani PF, Antia NH. Study of the evolution of nerve damage in leprosy. Part I-Lesions of the Index branch of the radial cutaneous nerve in early leprosy. Lepr India, 1980; 52: 5.

2 Shetty VP, Mehta LN, Irani PF, Antia NH. Study of evolution of nerve damage in leprosy. Part II-Observations on the Index branch of the radial cutaneous nerve in contacts of leprosy. Lepr India, 1980; 52, 19.

${ }^{3}$ Shetty VP, Vidyasagar PB, Antia NH. Study of evolution of nerve damage in leprosy. Part III-Sciatic nerve lesions in mice inoculated with $M$. leprae with nerve conduction velocity correlates. Lepr India, 1980; 52: 26.

4 Wager O. Immunological aspects of leprosy with special reference to auto-immune disease. Bull WHO, 1969; 41: 793. 
${ }^{5}$ Neta R, Salvin SB. Adjuvants in the Induction of Suppressor Cells. Infect Immunity, 1979; 23: 360.

6 Mshana R, Humber D, Harboe M, Belehu A. Nerve damage following intraneural injection of M. leprae into rabbits pre-sensitized to mycobacteria. Clin Exp Imm, 1983; 52: 441 .

7 Wright DJM, Hirst RA, Waters MFR. Neural autoantibodies in leprosy. Lepr Rev, 1975; 46: 157.

${ }^{8}$ Saida T, Saida K, Silberberg DH, Brown MJ. Transfer of Demyelination by intraneural injection of experimental allergic neuritis serum. Nature, 1972; 272: 639.

9 Crawford C, Evans D, Evans E. EAN induced by sensory nerve myelin may provide a model for non-lepromatous leprosy. Nature, 1974; 251: 223.

10 Boddingius J. Ultrastructural changes in Blood vessels of peripheral nerves in leprosy neuropathy. I. Tuberculoid and borderline-tuberculoid leprosy patients. Acta Neuropath (Berl), 1976; 35: 159. 